\title{
Prevalence of Insomnia and Internet Dependence Amidst the COVID 19 among the Northeast Indian Population: A Preliminary Study
}

\author{
Sanjenbam Yaiphaba Meitei ${ }^{1}$ (D) P. S. Vaveine $\mathrm{Pao}^{1} \cdot \mathrm{Kh}$. Dimkhoihoi Baite ${ }^{1} \cdot$ Henry Konjengbam $^{1}$
}

Received: 20 January 2021 / Revised: 10 July 2021 / Accepted: 12 July 2021 / Published online: 19 July 2021

(c) The Author(s), under exclusive licence to Springer Nature Singapore Pte Ltd. 2021

\begin{abstract}
Introduction COVID 19 pandemic lockdown affects normal daily activities making people vulnerable to sleep disorders, including insomnia and Internet addiction. The present study explores the prevalence of insomnia and Internet addiction amidst the pandemic while staying at home.

Methodology Data have been collected from 585 individuals of both sexes online through social media using a structured questionnaire that consists of insomnia severity and Internet addiction. Chi square and logistic regression analysis were done to associate the insomnia severity with Internet use behavior and addiction.

Results The overall prevalence of insomnia and subthreshold (mild) insomnia are $12.13 \%$ and $31.97 \%$, respectively, with females had insomnia more than males. Internet addiction correlates with insomnia as staying online more than initially intended increases insomnia significantly.

Conclusion COVID 19 pandemic lockdown affects sleep health as sleep-related disorders are on the rise, which could correlate to mental health. The study further seeks attention from researchers, health experts, and related stakeholders to develop a more holistic approach to uncover the problems and promote sleeping awareness.
\end{abstract}

Keywords Insomnia $\cdot$ COVID $19 \cdot$ Internet addiction $\cdot$ Pandemic $\cdot$ Awareness

\section{Introduction}

COVID 19 pandemic has created a massive impact on overall health worldwide, including mental health. Due to imposed restrictions on mobility, people remain confined to home with more time spent on smartphones or the Internet for leisure and work, disturbing the daily schedule, even affecting sleep health. Adequate sleep is essential for normal growth, which enables the hormone secretion required for normal physical development. In this pandemic, sleep problems such as insomnia, late bedtimes, or weird dreams have become a pattern [1] that is of utmost concern. Insomnia is one of the common sleep problems, with a prevalence of $8-40 \%$ in the general population [2]. Insomnia has consequences on cognitive and physical functioning, resulting in an emotional, social, and physical breakdown. Moreover, it also enhances the risk of cardiometabolic and

Sanjenbam Yaiphaba Meitei

sanyaimei@gmail.com; yaiphabameitei@manipuruniv.ac.in

1 Department of Anthropology, Manipur University, Imphal, Manipur 795003, India neurocognitive-related mortality and morbidity too [2]. Several studies have claimed that sleep disorders or insomnia being associated with extensive behavioral Internet use [3], which is more common among youths [4].

The situation can become more severe in this pandemic as more people, irrespective of being adolescents or children, are engaged in telecommunication mediums through their smartphones or laptop. Moreover, the government has encouraged e-learning and e-communication too. More extended access to e-communication or e-learning through work from home or online class models makes people more engaged in the Internet or online. Such excessive use of the Internet can cause severe Internet addiction problems, affecting socio-mental health during this pandemic. With this background, the present study attempts to understand the impact of Internet usage on insomnia among Northeast Indian adults amidst the pandemic. 


\section{Materials and Methods}

The present study was conducted online during May-June 2020. Five hundred eighty-five (585) individuals (both tribes and non-tribes) staying in and outside Manipur, Northeast India, of both sexes participated in the study. Manipur is a small state of India in the extreme northeastern part, adjoining South East Asia's international countries. Manipur, along with other northeastern states of India, has an Internet Penetration Rate (IPR) of 38\%, indicating $38 \%$ of its population are Internet users [5]. With this IPR, the proposed sample size is expected to give a Confidence Interval of $90 \%$, even in a large community [6]. A questionnaire, including the study's objective and other parameters, is created in the google form. Respondents were approached through social media, particularly Facebook and WhatsApp group or individuals. The survey link was sent and shared in different groups, as many as possible, without restricting only a few groups where the investigators are members, in the snowball sampling model. It was presumed that those who would respond had implied consent to participate in the study. The participants are in the age group of 18-50 years, having diverse professions including medicals, teachers, researchers, and architects. The questionnaire includes personal information, lockdown effect, Internet use behaviors of the respondents, Insomnia Severity Index (ISI), and Young's Internet Addiction Diagnostic Questionnaire (IADQ). Duplication of respondents was checked by restricting one-time submission of the google form and cross-checking email and name repetition. Those who have not given the details of personal information were excluded from the study. The questionnaire was prepared in the English language in a simple sentence with closed-ended questions (e.g., Yes/No/Not Sure) to make every respondent understand the questions. Translation of the questionnaire in the local language was not done as the study was targeted at the educated population of Manipur, especially students and professionals.

\subsection{Measures}

ISI is a brief instrument designed to assess the severity of both night-time and day-time insomnia components [7]. It is a 7-item self-report questionnaire with validation of $86.1 \%$ sensitivity and $87.7 \%$ specificity for detecting insomnia cases in the community sample. The Hindi translated version of ISI also holds reliability of 0.91 [8]. It is commonly used as a metric of treatment response in clinical research. Young's IADQ is an eight-item questionnaire based on DSM -IV diagnostic criteria of pathological gambling. Addiction is identified to at least five 'yes' to the questions [9].

\subsection{Statistical Analysis}

The prevalence of insomnia severity was calculated; Chi square was analyzed to correlate insomnia severity with Internet addiction and parameters. Chi-square analysis was calculated to test the significance of associated variables on insomnia. Cramer's V was also considered to determine the strength of associations in the significance test. Logistic regression analysis was done to predict the risk of insomnia severity in two different models: the bio-demographic variables and Internet use behavior during the lockdown are considered dependent variables in the first model, while IADQ Internet addiction parameters in the second model. In both models, insomnia severity status is the independent variable, and for convenient analysis, moderate insomnia and severe insomnia are put together as clinical insomnia. A multicollinearity test was done to check the possible correlation between the independent variables (predictors) that can be problematic in logistic regression analysis. Variance Inflation Factor (VIF) values of less than 5 with tolerance greater than 0.2 are considered the absence of multicollinearity [10]. Statistical analyses were done through SPSS version 26, at the significance level of 0.05 .

\section{Results}

The overall prevalence of clinical insomnia is $12.13 \%$ (moderate one $11.11 \%$ and severe insomnia with $1.02 \%$ ), and that of subthreshold insomnia is $31.97 \%$. Gender-wise prevalence of insomnia severity, as revealed in Table 1, shows that females had insomnia with $32.70 \%$ subthreshold, $11.79 \%$ moderate, and $1.14 \%$ severe clinical insomnia than males (subthreshold insomnia 31.37\%; moderate clinical insomnia: $10.56 \%$; severe clinical insomnia $0.93 \%$ ). Males are more Internet-addicted, with $29.19 \%$ than females $(27.76 \%)$. $9.63 \%$ of males are Internet-addicted as well as having subthreshold insomnia. Similarly, 9.89\% of females are having subthreshold insomnia and Internet addiction. Furthermore, $5.59 \%$ and $6.08 \%$ of males and females face moderate clinical insomnia and are prone to Internet addiction. Only $0.93 \%$ and $0.76 \%$ of the male and females have Internet addiction and severe clinical insomnia.

Chi square analysis (Table 2) shows a significant association of Internet addiction with insomnia severity in males, females, and in totality too $(p<0.05)$, with Cramer's $\mathrm{V}$ reaching up to 0.24 . Of the Internet used behaviors, time spent exposed to the Internet is statistically associated with insomnia $(p<0.05)$, with spending more than $6 \mathrm{~h}$ are more likely to have insomnia in both males and 
females, and together. While, watching movies online and increased accessed are significantly associated in males and combining both the genders $(p<0.05)$. Among Internet addiction parameters, longing for more time for Internet consumption and staying online longer than initially intended are also significantly associated with insomnia severity in aggregate and gender-wise $(p<0.05)$. Among the males only, Internet preoccupied, loss of control, and concealed use are significantly associated with insomnia. In contrast, withdrawal nature and risking behavior are significantly associated with insomnia severity among females. In the totality of males and females, all the addiction parameters are significantly associated with insomnia severity.

Table 3 presents logistic regression odd ratios of the studied variables concerning insomnia. It is observed that males are less likely to have insomnia, both subthreshold, and clinical, though it is not significant. Watching movies online, more time spent on the Internet in a day, and increased access during the lockdown are more likely to develop insomnia. Time spent on the Internet is statistically associated with clinical insomnia, as lesser time spent than $6 \mathrm{~h}$ is less likely to develop insomnia. Watching movies online is too significantly associated with subthreshold insomnia, and increasing the Internet access in both insomnia cases, though it is non-significant. In Internet addiction parameters, those who use the Internet longer than initially intended tend to have more insomnia significantly. Multicollinearity can be ruled out as the VIF value is below the cutoff (1.14-1.35 and 1.10-2.82) with collinearity tolerance higher than 0.2.

\section{Discussion}

Quality sleep is as essential to our health and well-being as adequate nutrition and exercise do. Insufficient and inadequate sleep can lead to several complications. Researchers give less importance to sleep problems than nutritional and other health-related issues, though it is one of the utmost necessary healthcare. A few studies have covered insomnia and sleep problems or related disorders from India [8, 11-15]. Moreover, the impact of COVID 19 lockdown on psychosocial health is well reported, including changes in sleep quality and sleep patterns $[14,16]$.

Insomnia is regarded as one of the leading sleep problems that affect nearly $10 \%$ of the general population [17], though its prevalence may vary from 8 to $40 \%$ [2]. It often goes undiagnosed and untreated, making the quality of life deteriorated and vulnerable [18]. It is often associated with psychological problems such as depression, anxiety, and lack of empathy [2, 14, 19-21], with more psychological disturbances led to insomnia, even during and pre-pandemic days. Screening of insomnia in the general healthy population is 
Table 2 Significance test and strength of Internet used behaviors and addiction parameters on insomnia severity

\begin{tabular}{|c|c|c|c|c|c|c|}
\hline \multirow[t]{2}{*}{ Studied variable } & \multicolumn{2}{|l|}{ Male } & \multicolumn{2}{|l|}{ Female } & \multicolumn{2}{|c|}{ In totality } \\
\hline & $\chi^{2}$ & Cramer's V & $\chi^{2}$ & Cramer's V & $\chi^{2}$ & Cramer's V \\
\hline \multicolumn{7}{|l|}{ Behaviors } \\
\hline Work from $($ Yes/No) & 4.22 & 0.115 & 1.44 & 0.074 & 1.91 & 0.057 \\
\hline Play online game (Yes/No) & 0.81 & 0.050 & 1.46 & 0.075 & 1.46 & 0.050 \\
\hline Watch movie online (Yes/No) & $9.02 *$ & 0.167 & 4.89 & 0.136 & $12.60 *$ & 0.147 \\
\hline Time spent $(2-4 \mathrm{~h} / 4-6 \mathrm{~h} />6 \mathrm{~h}$ a day $)$ & $32.61 *$ & 0.225 & $17.81 *$ & 0.184 & $42.58^{*}$ & 0.191 \\
\hline Increased access (Yes/No) & $21.34 *$ & 0.182 & 6.31 & 0.110 & $18.95^{*}$ & 0.127 \\
\hline \multicolumn{7}{|l|}{ Addiction parameters } \\
\hline Internet preoccupied (Yes/No) & $9.11 *$ & 0.168 & 3.72 & 0.119 & $9.74 *$ & 0.129 \\
\hline Need for more time (Yes/No) & $10.20 *$ & 0.178 & $13.35^{*}$ & 0.225 & $21.47^{*}$ & 0.192 \\
\hline Loss of control (Yes/No) & $19.55^{*}$ & 0.246 & 4.74 & 0.134 & $22.13 *$ & 0.194 \\
\hline Withdrawal (Yes/No) & 6.71 & 0.144 & $9.10 *$ & 0.186 & $14.29 *$ & 0.156 \\
\hline Staying online longer (Yes/No) & $15.74 *$ & 0.221 & $15.85^{*}$ & 0.245 & $29.95 *$ & 0.226 \\
\hline Jeopardizing or risking (Yes/No) & 7.27 & 0.150 & $22.21 *$ & 0.291 & $21.51^{*}$ & 0.192 \\
\hline Concealed use (Yes/No) & $15.45^{*}$ & 0.219 & 3.91 & 0.122 & $17.51^{*}$ & 0.173 \\
\hline $\begin{array}{l}\text { Escaping from problems or of reliev- } \\
\text { ing a dysphoric mood (Yes/No) }\end{array}$ & 2.50 & 0.088 & 5.92 & 0.150 & $7.98 *$ & 0.117 \\
\hline Internet addiction (Yes/No) & $20.27 *$ & 0.251 & $15.31 *$ & 0.241 & $34.65 *$ & 0.243 \\
\hline
\end{tabular}

limited [12]. Internet excessive use also impacts sleep behaviors and sleep patterns, even leading to insomnia [3]. A few studies have correlated sleep disturbances, including insomnia, with Internet addiction [3, 4].

The present findings reveal a potential alarming issue of insomnia. However, it is a preliminary study, and it will be too early to deduce before thorough research. The overall prevalence of $12.13 \%$ clinical insomnia and $31.97 \%$ subthreshold in the present study is alarming. This finding is much higher than the reported prevalence of insomnia (9\%) and that of subthreshold insomnia (30\%) [22]. Subthreshold insomnia is identified as having a score of 8-14 in the Insomnia Severity Index, developed by Morin et al.[7], who report frequency, persistence, or consequences of insomnia symptoms lower than those indicated by diagnostic criteria [21]. The higher prevalence of insomnia among females highlights gender variation to insomnia, as women have a higher risk of being owed to different reasons, including biological and genetic factors [23-25].

Various factors may influence sleep patterns, quality often leading to insomnia and other related disorders. Heavy Internet use and spending a longer time on the Internet affect sleep quality and lead to insomnia [26]. The association of Internet addiction with sleep disturbances among adolescents has already been established [26-29]. In the present study, too, spending more time on the Internet, i.e., more than $6 \mathrm{~h}$ in a day, watching movies online, and increased access to the Internet in the lockdown correlate with the insomnia severity. It is also revealed in regression analysis as those who respond Yes in watching movies online variable is 1.653 more likely to have insomnia significantly. Those who spent lesser time on the Internet considerably have a less odd ratio, thereby indicating that spending more time have a higher risk significantly.

The overall prevalence of Internet addiction is found to be $28.55 \%$, which is alarming. Internet addiction and its parameters, especially longing for more time and spending more time than initially intended, are statistically correlated with insomnia [26, 30]. Staying on the Internet longer than initially planned has 1.674 and 4.012 times likely to have subthreshold and clinical insomnia, respectively. This finding correlates with the association of spending more than $6 \mathrm{~h}$ on developing insomnia. It further suggests that Internet addiction parameters are more likely to enhance insomnia prevalence, which is also revealed in the regression analysis, mainly staying longer on the Internet than initially planned.

Sleep problems and insomnia can be severe on such pandemic days as more people suffer from anxiety, helplessness, and other mental problems. The study highlights the importance of sleep awareness, which has become an emerging issue for promoting quality and healthy sleep. The National Sleep Policy in India has been recommended, considering its importance in overall health consequences [31].

\section{Limitation}

The study is of its first kind in the northeastern part of India. The lack of comparable data from the pre-pandemic period from this region could be one of the limitations of the study. The data could not generate the severity of the sleep problems in general as it could be due to lockdown 
Table 3 Odd ratio risks of insomnia severity amidst the COVID 19 lockdown

\begin{tabular}{|c|c|c|c|c|}
\hline \multirow[t]{2}{*}{ Variable } & \multicolumn{2}{|c|}{ Subthreshold insomnia } & \multicolumn{2}{|c|}{ Clinical insomnia } \\
\hline & $\begin{array}{l}\text { OR CI } \\
(95 \%)\end{array}$ & $\begin{array}{l}\text { OR CI } \\
(95 \%)\end{array}$ & $\begin{array}{l}\text { OR CI } \\
(95 \%)\end{array}$ & OR CI $(95 \%)$ \\
\hline
\end{tabular}

Demographic and internet used behavior

\begin{tabular}{|c|c|c|c|c|}
\hline Age & 1.033 & $0.98-1.08$ & 0.984 & $0.91-1.06$ \\
\hline \multicolumn{5}{|l|}{ Gender } \\
\hline (Male) & 0.960 & $0.65-1.43$ & 0.907 & $0.51-1.62$ \\
\hline \multicolumn{5}{|c|}{ Occupational status $\$$} \\
\hline $\begin{array}{c}\text { (Work- } \\
\text { ing) }\end{array}$ & 0.845 & $0.54-1.32$ & 1.464 & $0.78-2.75$ \\
\hline \multicolumn{5}{|c|}{ COVID 19 lockdown affecting daily schedule } \\
\hline (Yes) & 1.607 & $0.64-4.00$ & 0.692 & $0.26-1.84$ \\
\hline (No) & 1.147 & $0.38-3.46$ & 0.336 & $0.07-1.59$ \\
\hline
\end{tabular}

Work from home during lockdown

$\begin{array}{lllll}\text { (Yes) } & 0.887 & 0.61-1.29 & 0.810 & 0.47-1.40\end{array}$

Play online game

$\begin{array}{llll}\text { (Yes) } \quad 0.936 & 0.63-1.39 & 0.987 & 0.55-1.76\end{array}$

Watch online movie

(Yes) $1.653^{*} \quad 1.01-2.69 \quad 1.320 \quad 0.63-2.78$

Time spent

$\begin{array}{lccll}(2-4 \mathrm{~h}) & 0.610 & 0.37-1.02 & 0.187^{*} & 0.09-0.40 \\ (4-6 \mathrm{~h}) & 0.673 & 0.40-1.12 & 0 . .429^{*} & 0.23-0.82 \\ \text { ncreased access } & & & \\ \text { (Yes) } & 1.029 & 0.54-1.97 & 1.272 & 0.46-1.41 \\ \text { (No) } & 0.577 & 0.27-1.24 & 0.722 & 0.20-2.62\end{array}$

IADQ Internet addiction

Internet preoccupied

(Yes) 1.380

$0.88-2.16 \quad 0.805$

$0.41-1.58$

Need for more time

(Yes) $\quad 1.516$

$0.98-2.35 \quad 1.403$

$0.73-2.70$

Loss of control

(Yes) 1.33

Withdrawal

(Yes) $\quad 0.871$

Staying online longer

(Yes) 1.674*

Jeopardizing or risking

$\begin{array}{lllll}\text { (Yes) } \quad 0.376 & 0.09-1.49 & 1.237 & 0.35-4.35\end{array}$

Concealed use

$\begin{array}{lllll}\text { (Yes) } & 1.087 & 0.64-1.84 & 1.608 & 0.82-3.17\end{array}$

Escaping from problems or of relieving a dysphoric mood

\begin{tabular}{lrrrr}
$($ Yes $)$ & 1.079 & $0.73-1.60$ & 1.492 & $0.82-2.71$ \\
Internet addiction & & & \\
(Yes) & 0.857 & $0.44-1.69$ & 1.276 & $0.47-3.46$ \\
\hline
\end{tabular}

${ }^{\$}$ For better comparison, occupation is divided into working and nonworking; the latter comprises students, job seekers, and homemakers

*Significance at 0.05

and excessive Internet use. Moreover, the offline mode of data collection can give a more detailed insight into sleep problems, including sleep hours and patterns, missing in the present study. Despite these certain limitations, the study puts forth new and vital yet often neglected health concerns.

\section{Conclusion}

Sleep awareness has become an emerging issue for promoting health as sleep problems affect overall health. Sleep problems and insomnia can be severe on such pandemic days as more people suffer from anxiety, helplessness, and other mental issues. The present study highlights one of the emerging health problems in this digital era. Sleep problems have been a common issue in the current modern lifestyle days. The prone to Internet addiction may enhance sleep-related disorders more endemic in the present studied population group. The question that arises here is that the lockdown imposition due to COVID 19 pandemic makes the problem more severe, challenging to answer as there are not enough data. Internet addiction and prolonged use may have contributed to insomnia in the studied populations. The study further seeks attention from researchers, health experts, and related stakeholders to look to the problems of sleep disorder and its consequences; and promote awareness to the public.

Acknowledgements We would like to thank all the individuals who participated in the study.

\section{Declarations}

Conflict of interest The authors declare that they have no actual or potential conflict of interest. There was no financial support for the study.

Ethical approval Due to the COVID-19 pandemic lockdown, ethical approval of the study was not taken. However, the study was conducted following the ethical standards of the institutional and/or national research committee and with the 1964 Helsinki declaration and its later amendments or comparable ethical standards.

Informed consent The participants were informed of the objectives of the study in the google form. Those who respond to the form were considered to have given consent. Personal information was disclosed to maintain the privacy of the participants.

\section{References}

1. Innocenti P, Puzella A, Mogavero MP, Bruni O, Ferri R. Letter to editor: CoVID-19 pandemic and sleep disorders-a web survey in Italy. Neurol Sci. 2020;41(8):2021-2.

2. Fernandez-Mendoza J, Vgontzas AN. Insomnia and its impact on physical and mental health. Curr Psychiatry Rep. 2013;15(12):418. 
3. Alimoradi Z, Lin CY, Broström A, Bülow PH, Bajalan Z, Griffiths $\mathrm{MD}$, et al. Internet addiction and sleep problems: a systematic review and meta-analysis. Sleep Med Rev. 2019;47:51-61.

4. Chen YL, Gau SSF. Sleep problems and Internet addiction among children and adolescents: a longitudinal study. J Sleep Res. 2016;25(4):458-65.

5. Keelery S. Internet penetration rate across India in 2019 by state [Internet]. 2020. https://www.statista.com/statistics/1115129/ india-Internet-penetration-by-state/

6. Mannocci A, Bontempi C, Giraldi G, Chiaradia G, de Waure C, Sferrazza A, et al. EpiInfo as a research and teaching tool in epidemiology and statistics: strengths and weaknesses. Ig Sanita Pubbl. 2012;68(1):85-96.

7. Morin CM, Belleville G, Bélanger L, Ivers $\mathrm{H}$. The insomnia severity index: psychometric indicators to detect insomnia cases and evaluate treatment response. Sleep. 2011;34(5):601-8.

8. Lahan V, Gupta R. Translation and validation of the insomnia severity index in hindi language. Indian J Psychol Med. 2011;33(2):172-6.

9. Young KS. Internet addiction: the emergence of a new clinical disorder. Cyberpsychol Behav. 1998;1(3):237-44.

10. Kim JH. Multicollinearity and misleading statistical results. Korean J Anesthesiol. 2019;72(6):558-69.

11. Shah N, Bang A, Bhagat A. Indian research on sleep disorders. Indian J Psychiatry. 2010;52(1):S255-9.

12. Panda S, Taly AB, Sinha S, Gururaj G, Girish N, Nagaraja D. Sleep-related disorders among a healthy population in South India. Neurol India. 2012;60(1):68-74.

13. Siddalingaiah HS, Chandrakala DSA. Sleep pattern, sleep problems and comorbidities among resident doctors at a tertiary care institution in India: a cross sectional study. Int J Community Med Public Heal. 2017;4(12):4477-84.

14. Gupta R, Grover S, Basu A, Krishnan V, Tripathi A, Subramanyam A, et al. Changes in sleep pattern and sleep quality during COVID-19 lockdown. Indian J Psychiatry. 2020;62(4):370-8.

15. Gupta R, Lahan V. Insomnia associated with depressive disorder: primary, secondary, or mixed. Indian J Psychol Med. 2011;33(2):123-8.

16. Grover S, Sahoo S, Mehra A, Avasthi A, Tripathi A, Subramanyan A, et al. Psychological impact of COVID-19 lockdown: an online survey from India. Indian J Psychiatry. 2020;62(4):354-62.

17. Pigeon WR. Diagnosis, prevalence, pathways, consequences \& treatment of insomnia. Indian J Med Res. 2010;131(2):321-32.

18. Benca RM. Diagnosis and treatment of chronic insomnia: a review. Psychiat Serv. 2005;56:94-102.

19. Shimizu T. Sleep habits and its relation to depression. Nihon Rinsho. 2012;70(7):1206-10.
20. Morin CM, Carrier J. The acute effects of the COVID-19 pandemic on insomnia and psychological symptoms. Sleep Med. 2021;77:346-7.

21. Cerolini S, Ballesio A, Lombardo C. Emotional experience, presence and severity of insomnia and depressive symptoms: an ecological study of their effects on sleep quality. Ment Health Fam Med. 2016;12:282-7.

22. Morin CM, LeBlanc M, Daley M, Gregoire JP, Mérette C. Epidemiology of insomnia: prevalence, self-help treatments, consultations, and determinants of help-seeking behaviors. Sleep Med. 2006;7(2):123-30.

23. Lind MJ, Aggen SH, Kirkpatrick RM, Kendler KS, Amstadter AB. A longitudinal twin study of insomnia symptoms in adults. Sleep. 2015;38(9):1423-30.

24. Mallampalli MP, Carter CL. Exploring sex and gender differences in sleep health: a society for women's health research report. J Women's Heal. 2014;23(7):553-62.

25. Li RHY, Wing YK, Ho SC, Fong SYY. Gender differences in insomnia - A study in the Hong Kong Chinese population. J Psychosom Res. 2002;53(1):601-9.

26. Younes F, Halawi G, Jabbour H, El ON, Karam L, Hajj A, et al. Internet addiction and relationships with insomnia, anxiety, depression, stress and self-esteem in university students: a crosssectional designed study. PLoS ONE. 2016;11(9):0161126.

27. Cheung LM, Wong WS. The effects of insomnia and Internet addiction on depression in Hong Kong Chinese adolescents: an exploratory cross-sectional analysis. J Sleep Res. 2011;20(2):311-7.

28. Jain A, Sharma R, Gaur K, Yadav N, Sharma P, Sharma N, et al. Study of Internet addiction and its association with depression and insomnia in university students. J Fam Med Prim Care. 2020;9(3):1700-6.

29. Tokiya M, Itani O, Otsuka Y, Kaneita Y. Relationship between Internet addiction and sleep disturbance in high school students: a cross-sectional study. BMC Pediatr. 2020;20(1):379.

30. Ekinci Ö, Çelik T, Savaş N, Toros F. Association between Internet use and sleep problems in adolescents. Noropsikiyatri Ars. 2014;51(2):122-8.

31. Akhtar N, Mallick H. Recommendations for a national sleep policy in India. Natl Med J India. 2019;32(1):59-60.

Publisher's Note Springer Nature remains neutral with regard to jurisdictional claims in published maps and institutional affiliations. 